\title{
Diseños participativos en el i-doc colaborativo: modelo de análisis y evaluación
}

\section{Participatory designs in the collaborative i-doc: a model of analysis and an evaluation}

\author{
Balaguer, J. ${ }^{1}$ \\ Recibido: 14-05-2020 - Aceptado: 10-08-2020 \\ https://doi.org/10.26441/RC19.2-2020-A3
}

RESUMEN: El ámbito del documental interactivo ha tenido desde sus inicios una vertiente colaborativa. En la presente investigación se propone un modelo de análisis de la participación de la comunidad online en este tipo de producción, elaborado a partir de la literatura previa sobre esta cuestión. Después, se ha analizado una muestra de casos que ha evidenciado la diversidad de formas que adquiere la contribución de la comunidad conectada. A partir de las distintas tendencias detectadas, se han identificado y descrito cinco diseños participativos con distintas intensidades y finalidades. Finalmente, el modelo se ha aplicado a cada uno de estos diseños para su evaluación, lo que permite concluir que una participación significativa tendría que combinar varios de estos diseños teniendo en cuenta tanto la articulación de la comunidad alrededor del documental como su incidencia en el significado.

Palabras clave: documental interactivo; participación cultural; creación colectiva; comunidad; interactividad; empoderamiento.

\begin{abstract}
The interactive documentary field has a collaborative dimension since its beginning. This research proposes a model for the analysis of the participation of online communities within interactive documentary, developed on the basis of the previous scientific literature on this topic. Then, analysed case studies have shown the diversity of forms regarding contribution from online communities. On the basis of the different trends observed, five participative designs with different intensities and purposes have been described. Finally, the model has been applied to each design for its evaluation, allowing us to conclude that a significant participation would have to combine several of these designs considering the articulation of participant communities around documentary as well as its contribution to documentary meaning.
\end{abstract}

Keywords: interactive documentary; cultural participation; collective creation; communities; interactivity; empowerment.

\footnotetext{
${ }^{1}$ Juanjo Balaguer es graduado en Comunicación Audiovisual, Máster en Nuevos Medios Interactivos y Periodismo Multimedia y Doctorando en el programa de Ciencias Sociales en la Universidad de Granada. balaguerjuanjo@ gmail.com, https://orcid.org/0000-0002-0776-5888
} 


\section{Introducción ${ }^{2}$}

Tanto la interactividad como la participación son características esenciales de la producción cultural contemporánea. A pesar de esta centralidad, no existe consenso en la definición del primer concepto, en parte debido a la diversidad de enfoques que lo abordan como objeto de estudio, según señalan Barry y Doherty (2016). Cuando se habla de interactividad, los autores indican que ha predominado un discurso sobre el empoderamiento de la audiencia, pero su experiencia variaría dependiendo de las diversas apreciaciones, modos y estrategias que se asocian a lo interactivo. Para otros autores, la interacción no sería en sí misma generadora de una emancipación del público, sino solo una condición de posibilidad para la participación, noción en la que sí podría residir ese empoderamiento (Carpentier, 2011; Jenkins y Carpentier, 2013).

Ha habido igualmente una dificultad persistente para definir la participación, abordada también desde diversas disciplinas (Jenkins, Ford y Green, 2013). El amplio abanico de prácticas que se han descrito como participativas en la era digital ha tenido como contrapartida una serie de análisis críticos, ya sea por la apropiación del trabajo creativo de las comunidades conectadas por parte de las empresas (Deuze, 2008), por los discursos sobre la democratización tras la Web 2.0 que no tenían en cuenta por las condiciones materiales de esta participación (Van Dijck, 2009), o por la visión determinista según la cual la tecnología, por sí misma, daría lugar a efectos sociales positivos (Marwick, 2019). En este sentido, Carpentier (2011) advertía de la necesidad de definir distintas intensidades en la participación, así como de tener en cuenta que la etiqueta "participativo" se asocia a prácticas que no lo son, mientras que Jenkins reconocía que el término "cultura participativa" se empleaba con superficialidad, incluso cuando en realidad no se otorgaba ningún control al usuario (Jenkins y Carpentier, 2013).

El establecimiento de una cierta equivalencia entre participación, interacción y acceso sería una consecuencia de esa percepción laxa de la primera, según Carpentier (2015). Esto daría lugar a la pérdida de una participación significativa, cuyo componente definitorio, para el autor, es el poder. Por el contrario, si se incide en la distinción entre estos tres conceptos, las relaciones de poder y la toma de decisiones podrían ser los elementos clave para evaluar los procesos comunicativos considerados como participativos (Carpentier, Duarte y Ribeiro, 2019). Del mismo modo, Nash (2014a) indica que, aunque la interactividad modifica el estatuto de la audiencia e interviene en su relación con los demás agentes del proceso comunicativo, esto no significa que implique su empoderamiento.

La autora realiza un análisis de la participación y de la interactividad desde un enfoque social y en aplicación al documental interactivo o $i$-doc. En su perspectiva sobre el primer concepto, este no es mera interacción, sino que se traduce en la apertura de vías de negociación de los significados transmitidos gracias a la contribución de la comunidad. Tendría lugar entonces un reparto de poder entre el agente productor y la audiencia, lo que podría conllevar un incremento del pluralismo. En esa línea, muchos documentales interactivos pretenden generar un impacto social positivo, como han señalado diversos autores (Moreno y Gifreu-Castells, 2016; Rose, 2017b; Vázquez-Herrero y Moreno, 2017). Se ha destacado además la capacidad de los $i$-docs para activar la participación ciudadana (Cortés-Selva y Pérez-Escolar, 2016).

Aston y Odorico (2018) han resaltado también la capacidad de los documentales interactivos para generar discursos plurales, representar múltiples realidades y mostrar más perspectivas que la crea-

\footnotetext{
2 Este trabajo ha sido realizado en el marco del proyecto I+D (Ref. CSO2017-85965-P) "Transmedialización y Crowdsourcing en las narrativas de ficción y no ficción audiovisuales, periodísticas, dramáticas y literarias" (20182020) del Plan Estatal de Investigación (FEDER/Ministerio de Ciencia e Innovación - Agencia Estatal de Investigación, España).
} 
ción audiovisual lineal. Muchas producciones se fundamentan en una reivindicación de la diversidad que se traduce precisamente en la construcción del documental mediante la aportación de contenido por parte de la comunidad, con frecuencia a modo de autorrepresentación. Esta erosión de las fronteras entre roles habría convertido a la comunidad participante no solo en productora, sino también en protagonista del proceso comunicativo, lo que adquiere especial relevancia con respecto a la apertura de procesos distintivos de representación cultural.

De esta manera, son muchas las iniciativas que han realizado un discurso plural con vocación social gracias a su concepción participativa. El documental interactivo es un caso paradigmático porque, como recuerda Nash (2014c, 2017), la no ficción ha tenido siempre una función social que ahora los formatos recientes heredarían. Junto a esta tradición del documental, se encontraría la memoria de prácticas fílmicas de décadas anteriores que, a través de la participación, abrieron nuevos cauces en la producción cultural con la finalidad de generar representaciones diversas y herramientas más democráticas para tender así a un cambio social (Mateos y Gaona, 2015; Villanueva, 2015; Sucari, 2020).

Tanto el documental interactivo como los procesos participativos en la creación cultural son fenómenos de creciente relevancia y frecuente convergencia. Por ello, esta investigación propone un modelo de análisis de la participación en el $i-d o c$, según el sentido que se ha descrito en esta introducción. Después, el estudio de una muestra de casos permitirá describir distintos diseños participativos que son implementados en el documental interactivo. Seguidamente, estos serán evaluados mediante el modelo propuesto.

El objetivo principal de esta investigación es analizar la función social de la participación en este ámbito de la producción cultural, teniendo en cuenta la variedad de formas que acoge. Además, frente a la multitud de sujetos a los que puede referirse la participación, este artículo se centra en la comunidad en línea, siendo esta la principal receptora de la participación que se habilita en estos documentales.

\section{Elementos de la participación en la creación documental interactiva}

\subsection{El documental interactivo y su vertiente colaborativa}

Una revisión de la investigación sobre el documental interactivo nos muestra la existencia de múltiples definiciones. Encontramos así propuestas como la de Gifreu-Castells (2013), para quien el documental interactivo recoge las modalidades de representación de la no ficción convencional más otras nuevas a las que denomina modalidades de navegación e interacción, que implican distintas intensidades de participación en un determinado i-doc. Por otro lado, la definición de Odorico (2015) también incide en los distintos grados de participación.

Destacamos la definición de Aston y Gaudenzi (2012), dado que su amplitud resulta práctica para el estudio de un concepto que muestra una significativa diversidad en su forma, en su propósito o en las técnicas que emplea para su producción. Según las autoras, un documental interactivo o $i$-doc es "any project that starts with an intention to document the 'real' and that uses digital interactive technology to realize this intention can be considered an interactive documentary" (p. 126).

Más allá de la definición del $i$-doc, son numerosas las investigaciones que profundizan tanto en la interactividad como en la participación, teniendo en cuenta los distintos grados de intensidad que se proponen. El análisis del marco en que se producen la interactividad y la participación es fundamental, según Nash (2014c), para evaluar la función social de este tipo de producción. 
La participación se ha entendido frecuentemente como una categoría incluida dentro de la interactividad, por lo que se concebiría como una interactividad de alta intensidad. Así queda implícito en la noción amplia de lo interactivo por parte de Odorico (2015), que no solo haría referencia a acciones como elegir entre varias opciones predeterminadas que aparecen en la interfaz, sino también a procesos de creación colaborativa. No obstante, Gaudenzi (2013) advierte de que todas estas producciones son interactivas, pero no todas son participativas. Remarcando la frontera entre interacción y participación, la autora elabora una clasificación de la interactividad donde distingue entre los modos conversacional, hipertextual, experiencial y participativo (Aston y Gaudenzi, 2012; Gaudenzi, 2013). En este último, la tarea del equipo del documental consistiría — frente a su tradicional función de autoría - en establecer el escenario de una conversación con la comunidad participante (Aston y Gaudenzi, 2012). Esta última comenzaría entonces a formar parte del proceso productivo.

Nash (2012) también ha diferenciado entre tres estructuras de la interactividad: narrativa, categórica y colaborativa. El documental interactivo con estructura colaborativa pone en primer plano las aportaciones de la comunidad, que pueden tener lugar de diversas maneras.

Por último, Gifreu-Castells (2013) ha señalado distintas modalidades de navegación e interacción, como se indicó previamente. Las segundas aludirían a los procesos de participación que tienen lugar en el documental interactivo. Entre ellas, se encontraría la "interacción generativa", que se caracteriza porque la comunidad contribuye con su propio contenido.

Como ha ocurrido con la noción de participación en términos generales, su aplicación al ámbito del documental interactivo ha originado una serie de análisis que problematizan este fenómeno, especialmente cuando las producciones tienen una proyección social. La crítica se centra sobre todo en valorar si se habilita a la comunidad para influir en el discurso del documental. Hay que precisar que en un $i-d o c$, incluso cuando no incorpora participación, se cede parte del control narrativo a cambio de la interactividad (Gifreu-Castells, 2013). En su vertiente colaborativa, esta pérdida de control a favor del usuario se podría entender también como una distribución de la autoría.

Indagar en la función social del $i-d o c$ colaborativo requiere, para Nash (2014c), estudiar las relaciones que este genera, en especial con respecto a la comunidad. En este sentido, habrá que tener en cuenta su contribución a la voz del documental, concepto que la autora toma de Nichols (1983). Con ello, traslada la participación desde un espacio de contribución hasta otro de autoría. También advierte de la necesidad de distinguir entre "user actions that impact on the documentary text and the ability of users to engage with others through documentary" (p. 383). Esta distinción nos servirá de referencia para enumerar las complejidades que, según se ha argumentado, tendría que superar el documental interactivo para hacer más efectiva su función social.

\subsection{La comunidad en el documental interactivo}

Aunque se podría hablar de una autoría compartida en el documental interactivo gracias a la colaboración de la comunidad, la tradicional función de autor habría permanecido prácticamente inmutable (Gaudenzi, 2014). En términos similares, Aston y Odorico (2018) exponen el desequilibro que habría en las relaciones de poder entre el equipo de producción y la comunidad, por lo que un documental interactivo donde se reparta el control de manera simétrica sería todavía improbable.

Los límites que impiden alcanzar ese equilibrio son diversos. En ocasiones se ha señalado la forma que adopta la participación. En este sentido, se han empleado las categorías de "participación ejecutiva" (executory participation) y "participación estructural" (structural participation), descritas por Green, Bowen, Hook y Wright (2017). En los i-docs, se suele habilitar solo la primera categoría, esto es, contribuir en el contenido del documental. Pero se sostiene que, para crear una obra plural con la comunidad, sería imprescindible implementar también la segunda categoría, es decir, una 
participación que afectara a la estructura del documental. Para Gaudenzi (2013, 2014), compartir el control de la interfaz implicaría un nuevo grado de poder para la comunidad, e incluso un acto político. Por ello, contempla como conflictivo el hecho de que la participación se resuma con frecuencia en la aportación de contenido de la comunidad, porque esto connotaría que "the individual has a voice but should not have control on how it is used" (p. 238).

Se han señalado otros elementos a tener en cuenta para implementar una participación efectiva, como serían la capacidad de la comunidad para colaborar en la definición conceptual del documental o la etapa del proceso de producción en que tiene lugar la participación (Gaudenzi, 2014). En relación con la primera cuestión, Nash (2012) ha mencionado también la conveniencia de que los participantes puedan desafiar la perspectiva que toma el documental. Con respecto al segundo punto, parece incuestionable que si la comunidad colaborara en la definición inicial del proyecto, su aportación adquiriría nuevos matices, consiguiendo tener probablemente una mayor repercusión en el conjunto del documental.

Todo ello apunta a un cierto cuestionamiento en relación con la capacidad del $i$-doc colaborativo para dotar a la comunidad de una influencia significativa en la construcción de significado y equilibrar así la relevancia de los distintos agentes que forman parte del proceso creativo. En este sentido, parece que si una alta intensidad en la interactividad sería equivalente a la participación, una alta intensidad de la participación equivaldría a la coautoría.

A pesar de ello, hay que destacar que no todos los $i$-docs colaborativos pretenden desarrollar esa faceta participativa con una alta complejidad o dotar a la comunidad online de tanta responsabilidad. Así, incluso si la colaboración es asimétrica, puede ser productiva para la comunidad, como ha indicado Rose (2011). Más allá de la intensidad de la colaboración, habría que atender a las condiciones según las cuales el equipo de producción y la comunidad acuerdan la participación, sobre todo si se quiere evitar que el efecto positivo de esta quede mermado. Dovey (2014) emplea el concepto de framing, que define como "the process through which collaboration and participation is invited in the first place" (p. 22). Con ello se reivindica el desarrollo de un protocolo cuya aplicación explicite los términos en que se produce la colaboración. De esta manera, se podría preservar el valor que la creación colaborativa puede tener para la comunidad, incluso aunque no haya un equilibro de poder entre los distintos roles. La aplicación de este principio es particularmente relevante en aquellas iniciativas en las que la participación requiera de una labor creativa y de una fuerza de trabajo.

Además de la relación entre el equipo productor y la comunidad, también se pone atención a las interacciones que tienen lugar entre los miembros que forman la segunda. Su proceso de articulación parece esencial si se busca que la iniciativa incremente su proyección social. En este sentido se destaca, por un lado, que el equipo creativo del documental debe habilitar vías para facilitar la conexión y el diálogo entre los miembros de la comunidad (Nash, 2012). Por otro lado, Nash (2014b) advierte de la importancia de definir un tema de interés compartido entre participantes para motivar su implicación con la iniciativa y reforzar una cierta continuidad en su respuesta.

Finalmente, tras tomar una serie de prácticas colaborativas previas como referencia, Katerina Cizek expande la noción de la comunidad online al fijarse en organizaciones comunitarias que, gracias a procesos participativos mediáticos, se movilizan y llevan a cabo acciones fuera de las pantallas (en Rose, 2017a). De manera similar, Nash (2014c) destaca que las relaciones que se originan durante el proceso creativo pueden dar lugar a interacciones que trasciendan la producción. Así, la evaluación de la participación en el ámbito del $i$-doc no tiene solo en cuenta la influencia de la comunidad en el contenido, sino también su capacidad para generar procesos colectivos que sobrepasen el marco del documental interactivo. 


\section{Metodología}

La presente investigación propone un modelo para la evaluación de la participación de las comunidades online en los documentales interactivos. La revisión bibliográfica ha permitido obtener una base teórica sobre el documental interactivo y sobre la creación colaborativa, tanto en general como en su aplicación específica a esta modalidad de producción. Gracias a esta revisión se han detectado una serie de criterios que distintos autores han destacado para valorar si se produce una participación significativa de las comunidades online en el $i$-doc colaborativo, como ya se ha reflejado en el marco teórico. Esto ha servido como referencia para la elaboración de un modelo de análisis de la participación. Se ha dado especial atención a la distinción de Nash (2014c), antes citada, según la cual el estudio de la participación debe tener en cuenta tanto la influencia de la comunidad en el documental como las interacciones sociales que se originan gracias a su desarrollo.

Ambas cuestiones constituyen las categorías principales de este análisis: por un lado, la incidencia de la comunidad en la construcción de significado del documental; por otro, la articulación de la comunidad. Con la primera, se podrá valorar en qué medida el equipo productor y la comunidad comparten un cierto control sobre el contenido, es decir, qué impacto tiene en el documental la voz de quienes participan. La segunda permitirá atender a los procesos de acción colectiva que se generan gracias a la creación colaborativa.

Tras la identificación de estas categorías, se han escogido una serie de variables que servirán para medirlas. Estas variables tienen, como referencia, las contribuciones de la literatura científica que se han mencionado previamente. Con ello, el modelo considerará los siguientes puntos:

1. Articulación de la comunidad:

a) Activación colectiva. Se tendrá en cuenta si se fomenta una toma de conciencia colectiva o incluso nuevas vías de acción que puedan trascender el proceso de producción del documental. También se advertirá si la iniciativa cuenta en su fundamentación con algún tema de interés compartido que pueda favorecer vínculos entre miembros de la comunidad, así como su implicación con la iniciativa.

b) Apertura de procesos de diálogo que permitan crear o reforzar lazos colectivos.

2. Incidencia de la comunidad:

a) Carácter central o periférico de la participación.

b) Grado de influencia sobre el contenido. Se determinará valorando la relevancia de la contribución en el conjunto de la iniciativa, así como si la comunidad ha podido influir en la definición de la misma. También se tendrá en cuenta si su participación puede originar alternativas a los planteamientos del documental, desafiándolos, o por el contrario, solo puede reafirmarlos.

El siguiente paso de la investigación ha sido la selección de una muestra de casos para su estudio. Los casos se han escogido teniendo en cuenta su carácter colaborativo y su relevancia. Para estimar su relevancia, se ha procedido a realizar una búsqueda en repositorios, catálogos de productores y festivales de referencia.

El proceso ha comenzado con una consulta en la Docubase del MIT Open Documentary Lab, que es uno de los principales directorios de producciones de no ficción interactiva. Para tener en cuenta solo las iniciativas participativas, se ha realizado una búsqueda con los filtros "participatory", "co-creation", "community collaboration", “community generation", "collaborative storytelling” y "crowdsourcing". Además, para que la investigación tenga una perspectiva reciente, se han incluido solo los proyectos iniciados en el periodo entre 2014 y 2019. La selección, teniendo en cuenta solo 
aquellos casos en los que la participación se refería a la comunidad online, ha sumado nueve producciones (Life on Hold; Proyecto Quipu; Man on Bridge; Jerusalem, We Are Here; The Counted; Question Bridge: Black Males; Radio Right Left; The G Word: Transforming gender norms, one story at a time; y Primal). La muestra se ha completado después con una búsqueda en el listado de proyectos destacados por el Co-Creation Studio del MIT Open Documentary Lab (The Whiteness Project, (des)iguales), en InterDOC (Barnacas), en festivales de relevancia como IDFA (My Inner Wolf) y en el catálogo de un productor de referencia como es el National Film Board of Canada (The Space We Hold).

El análisis de la muestra ha llevado a identificar distintos diseños de la participación en función de su finalidad, su intensidad y la manera en que se estructura en la interfaz de los proyectos. Tras identificar aquellas tendencias que estaban presentes en varios casos, se ha procedido a describir cinco diseños de participación. Por último, se ha aplicado el modelo para evaluar el proceso participativo en cada uno de los diseños, obteniendo con ello conclusiones de conjunto sobre los casos analizados.

\section{Resultados}

\subsection{Categorización de diseños del proceso participativo en los documentales interactivos}

La muestra evidencia cómo estas producciones encuentran una amplia variedad en la forma que adquiere la participación, aunque todos los casos coinciden en que se dirigen a la comunidad online e intentan dar lugar a una relación cooperativa con los usuarios. Así, todos tienen componentes que trascienden la interacción, aunque no puede dejar de mencionarse que algunos presentan más bien mecanismos que se encuentran en la frontera entre interactividad y participación. Hay que incidir en que la etiqueta de participativo en un documental no siempre implica que la finalidad del equipo de producción sea establecer altas cotas de participación, como se ha indicado previamente. Y, además, la participación puede tener distintos fundamentos, como establecer un espacio de solidaridad, más allá de habilitar una vía para la construcción de un relato por parte de las personas participantes o de activar una comunidad. Por ello, conviene distinguir entre distintos diseños participativos en el documental interactivo.

Con esa finalidad, la presente investigación aporta una categorización original de algunos de los diseños participativos que tienen lugar en los $i$-docs colaborativos, lo que podrá ser empleado para definir y evaluar el papel de la comunidad conectada en este tipo de producción. No se trata de incluir todas las posibles formas que puede adoptar la participación en este tipo de producción, sino de describir una serie de tendencias que se han detectado en diversos casos. Hay que señalar además que estos diseños no son excluyentes, sino que pueden convivir en una misma iniciativa. Las categorías que proponemos son:

a) Participación testimonial. En este diseño, las iniciativas incluyen un espacio donde la comunidad puede hacer una aportación para mostrar su solidaridad o compromiso con otras personas cuya historia forma el centro de la narración. La participación tiene como finalidad la implicación en la historia, así como fomentar la empatía y un cierto vínculo con determinados colectivos. En algunos casos, la contribución se ubica en un espacio donde se reúnen todos los testimonios, a modo de mural, como ocurre en The Space We Hold o en Life on Hold. También encontramos este tipo de participación en Proyecto Quipu o en My Inner Wolf. En este último caso, el proceso consiste en subir una imagen personal para favorecer la identificación con el colectivo al que se dirige la iniciativa. 
Tabla 1. Casos de estudio y diseños participativos

\begin{tabular}{|c|c|c|c|c|}
\hline Título & Fecha & Autor/a & $\begin{array}{c}\text { Diseño } \\
\text { participativo }\end{array}$ & $\begin{array}{c}\text { Enlace (consultado el } \\
20 / 07 / 2020)\end{array}$ \\
\hline The Space We Hold & 2017 & $\begin{array}{l}\text { Tiffany Hsiung, Christopher } \\
\text { Kang y Patricia Lee }\end{array}$ & Testimonial & http://spacewehold.nfb.ca/ \\
\hline Life on Hold & 2015 & $\begin{array}{c}\text { Dima Shaibani, Ralph Dfouni, } \\
\text { Reem Haddad }\end{array}$ & Testimonial & $\frac{\text { http://lifeonhold.aljazeera.com/\#l }}{\text { en/loading }}$ \\
\hline Proyecto Quipu & 2015 & $\begin{array}{c}\text { Maria Court, Rosemarie } \\
\text { Lerner }\end{array}$ & $\begin{array}{l}\text { Testimonial y } \\
\text { expansiva }\end{array}$ & $\frac{\text { https://interactive.quipu-project. }}{\text { com/\#/en/quipu/intro }}$ \\
\hline My Inner Wolf & 2019 & Luna Maurer, Roel Wouters & Testimonial & https://myinnerwolf.nl/ \\
\hline Man on Bridge & 2014 & Ciaran Deeney & Documental & http://www.manonbridge.ie/ \\
\hline $\begin{array}{c}\text { Jerusalem, We Are } \\
\text { Here }\end{array}$ & 2016 & Dorit Naaman & Documental & https://jerusalemwearehere.com/\#l \\
\hline The Counted & 2015 & $\begin{array}{c}\text { Jamiles Lartey, Jon Swaine y } \\
\text { Oliver Laughland }\end{array}$ & Documental & $\begin{array}{l}\frac{\text { https://www.theguardian.com/us- }}{\text { news/ng-interactive/2015/jun/01/ }} \\
\frac{\text { the-counted-police-killings-us- }}{\text { database }}\end{array}$ \\
\hline Barnacas & 2018 & $\begin{array}{l}\text { Oscar Dhooge y Jesús } \\
\text { Martínez }\end{array}$ & $\begin{array}{l}\text { Dialógica y } \\
\text { expansiva }\end{array}$ & http://www.barnacas-webdoc.com/ \\
\hline Whiteness Project & 2016 & Whitney Dow & Dialógica & https://whitenessproject.org/ \\
\hline $\begin{array}{l}\text { Question Bridge: } \\
\text { Black Males }\end{array}$ & 2014 & $\begin{array}{c}\text { Chris Johnson, Hank Willis } \\
\text { Thomas, Bayete Ross Smith } \\
\text { y Kamal Sinclair }\end{array}$ & Generativa & http://questionbridge.com/ \\
\hline Radio Right Left & 2017 & Halsey Burgund & Generativa & https://radiorightleft.org/ \\
\hline (des)iguales & $\begin{array}{l}2015- \\
2016\end{array}$ & $\begin{array}{l}\text { Fernando Irigaray (Cátedra } \\
\text { Latinoamericana de } \\
\text { Narrativas Transmedia) }\end{array}$ & $\begin{array}{l}\text { Generativa y } \\
\text { expansiva }\end{array}$ & http://www.des-iguales.com/ \\
\hline The G Word & 2015 & $\begin{array}{c}\text { Breakthrough, Ishita } \\
\text { Srivastava }\end{array}$ & Generativa & $\frac{\text { https://us.breakthrough.tv/ }}{\text { thegword/ }}$ \\
\hline Primal & 2014 & Caroline Hayeur & Expansiva & http://primal.nfb.ca/en \\
\hline
\end{tabular}

Fuente: elaboración propia

b) Participación documental. Siguiendo la lógica de archivo que tienen muchos documentales interactivos, con este diseño se invita a la comunidad a que aporte material relacionado con lugares, individuos o sucesos. Se complementa así una base de datos, con frecuencia de carácter patrimonial, que gracias a su construcción creativa mediante el documental interactivo podrá ofrecer una experiencia al usuario en su acercamiento a la memoria de un colectivo o de un espacio recreado. En Man on Bridge, se reúne material para crear un archivo alternativo de la ciudad de Dublín y anima a la comunidad a que contribuya con fotografías. En Jerusalem, We Are Here, que cuenta la historia de un barrio de la ciudad a través del testimonio de palestinos cuyas familias fueron expulsadas, también se solicita información a la comunidad, así como imágenes o vídeos del barrio, con la finalidad de preservar una memoria colectiva del lugar. Por último, citamos The Counted, una iniciativa con una finalidad diferente, ya que su base de datos habilita la participación ciudadana para registrar información sobre personas asesinadas por la policía en los Estados Unidos. En este caso, la colaboración se inicia también como instrumento para la memoria pero, sobre todo, para generar una plataforma de denuncia e investigación.

c) Participación dialógica. Se trata de diseños que buscan la conversación entre los miembros de la comunidad, ya sea para que cuenten sus propias experiencias o para que compartan su 
posicionamiento sobre los temas examinados en el documental. Es el caso de Barnacas o de Whiteness Project, donde se habilitan foros de debate que complementan el resto de la iniciativa. En el primer caso, el foro colaborativo sirve también para obtener material de archivo relativo al tema del documental. En el segundo caso, el foro permite una conversación sobre cuestiones generales pero también sobre la aportación de cada una de las personas que protagoniza el documental. Al abrir una conversación, este diseño puede incidir en la formación de una comunidad y requiere, más que en otros casos, de un tema de cierto interés compartido para favorecer su implicación.

d) Participación generativa. La comunidad produce material expresamente para la iniciativa, siendo este el resultado de una elaboración creativa o de la expresión de experiencias personales que incorporan nuevas narrativas al documental. En algunos casos, la unión de todos los materiales aportados por la comunidad será lo que construya la obra, por lo que la participación es esencial para la culminación de la misma. Algunas de las iniciativas que presentan este diseño participativo son Question Bridge: Black Males, Radio Right Left, (des)iguales, The G Word: Transforming gender norms, one story at a time. Una de sus diferencias con respecto a la participación documental radica en que, en aquel caso, se trata de aportar material preexistente, ya sean datos, documentos textuales o archivos audiovisuales.

e) Participación expansiva. Este diseño da lugar a formas de participación que, aunque siguen vinculadas con el documental, lo sobrepasan. En algunos casos, el documental se origina como parte de un proyecto participativo más amplio o, al contrario, este último se origina como parte de un proceso de expansión del documental interactivo. Este diseño está vinculado a la transmedialidad. Recordamos que el documental interactivo ha sido señalado como formato nativo transmedia (Gifreu-Castells, Sánchez-Castillo y Galán, 2019). En otras ocasiones, la participación expansiva significa solo la habilitación de vías que pretenden provocar la reacción de la comunidad para que tome la iniciativa en una determinada causa, como la invitación en Proyecto Quipu a que el participante firme una petición. La participación expansiva está presente además en (des)iguales, que impulsa actividades paralelas como proyecciones en la calle, o en Primal, que invita a emplear su dinámica de participación como recurso educativo para su aplicación en las aulas y ofrece materiales para ello.

\subsection{Aplicación del modelo de análisis y evaluación de los diseños participativos}

Tras identificar las iniciativas que forman la muestra de estudio con los diseños participativos propuestos, se aplica a continuación el modelo para evaluar el proceso de participación:

a) Participación testimonial.

Tras dar a conocer o incidir en un conflicto que afecta a un determinado colectivo, los documentales que tienen un diseño de participación testimonial promueven la articulación de una comunidad online alrededor del tema del documental. Este diseño no parece suficiente para estimular la organización de la comunidad más allá de la obra interactiva, porque no se establecen vías de comunicación entre sus miembros ni se definen objetivos en común. Esta carencia dificultaría una relación productiva entre los miembros de la comunidad conectada. Sin embargo, sí requiere de implicación por parte de quienes participan, que podrán tomar conciencia de la extensión de un conflicto, lo que es consustancial a este diseño porque, sin ello, la comunidad no estaría impelida a participar. En este sentido, el proceso de concienciación colectiva favorecería una experiencia compartida que podría reforzar el carácter comunitario de la iniciativa.

Por otro lado, la incidencia de la comunidad en el documental es nula. Así, la participación es periférica, porque se construye como respuesta a una narración previa. Esto implica además que la 
comunidad no es partícipe en la definición del proyecto. Por todo ello, hay que destacar que, entre todos los diseños participativos, este es el más limitado.

b) Participación documental.

La comunidad online se forma alrededor de un tema sobre el que se solicitan datos, fotografías, vídeos e incluso historias personales. Su acción se produce, entonces, en el marco de un interés compartido cuya descripción es decisiva para motivar la participación. Si bien se puede originar un vínculo entre los miembros de la comunidad gracias a esa fundamentación, no se favorece que la acción de la comunidad experimente un trasvase a otros ámbitos, quedando así limitada a la construcción de la base de datos que articula la iniciativa. Tampoco se alienta un proceso de diálogo porque, en este diseño, la participación no tiene como finalidad la apertura de un debate sino más bien la realización de una tarea.

El grado de influencia que ofrece este diseño participativo es medio. Por un lado, la comunidad colabora en la construcción de la iniciativa, lo que en este caso puede producirse de manera periférica o central; por otro lado, la definición del proyecto se habría establecido previamente. Además, no cabría un posicionamiento, por parte de quienes participan, que pudiera desafiar ese relato porque, frente a diseños participativos que permiten un margen más amplio para ofrecer una interpretación diferente o una propuesta creativa alternativa, la participación documental tiene una mayor orientación fáctica.

c) Participación dialógica.

La participación dialógica puede dar lugar a procesos colectivos significativos al poner en relación de forma directa a los miembros de la comunidad. Así, tanto la apertura de un espacio de debate como la propuesta de un tema de interés compartido podrían facilitar la construcción colectiva de conocimiento gracias a la interacción. Por ello, este es un diseño apropiado para conseguir una cierta activación comunitaria.

Esto no impide que el grado de influencia de la comunidad sea medio, teniendo en cuenta además que este diseño participativo es periférico. Aun así, un espacio de diálogo es también una vía para una comunicación disruptiva y para la expresión de voces discrepantes. Por este motivo, aunque la participación dialógica ocupe un espacio marginal, e incluso sin dejar su marca en el conjunto del documental, su presencia puede generar una contribución significativa desde el espacio que ocupa la comunidad conectada.

d) Participación generativa.

Se trata del diseño participativo que propone una mayor creatividad a la comunidad, que actúa movida por la definición de un tema que la interpela personalmente o le causa interés creativo. Por ello, la configuración de un relato colectivo que favorece la participación ocupa un elemento central en la construcción comunitaria que favorece este diseño participativo. En este sentido, el contenido de la comunidad forma parte de un relato, pero no suele originar un debate porque todas las aportaciones pertenecen a un conjunto más bien homogéneo, no siendo puestas entre sí en relación directa, como sí ocurre con la participación dialógica, lo que limitaría la posibilidad de una conversación.

La contribución de la comunidad podrá tener un carácter periférico o central. Así, son frecuentes los documentales interactivos basados, sobre todo, en este tipo de aportación por parte de los miembros de la comunidad. Aunque su grado de incidencia sobre el contenido es elevado, es aquí donde más pertinencia adquiriría la reivindicación de una creación colectiva que permitiera también una participación estructural, según se ha descrito con anterioridad. En la libertad creativa que se le concede, la comunidad puede encontrar espacios de propuesta y de expresión alternativa al discurso mantenido en el documental. 
e) Participación expansiva.

En este diseño la participación ya no guarda tanta relación con la influencia de la comunidad sobre el contenido del documental, como con su conformación como grupo. Propone al $i$-doc como espacio nuclear de un encuentro que después continuará en otros espacios y que, a veces, abandona lo virtual. La participación dirigida a la comunidad online a través del documental sirve de catalizador para la generación de una acción colectiva más amplia, por lo que este diseño participativo encuentra su fundamento en procesos de diálogo e intereses compartidos que colaboran en la consecución de esa perspectiva colectiva.

Todo ello no significa, como se ha indicado, que la incidencia de la comunidad en el documental interactivo sea particularmente significativa. La participación adquiere aquí otro sentido, más bien relacionado con la capacidad de la no ficción para generar procesos colectivos. Por este motivo, la participación de la comunidad ocupa un lugar periférico en relación con el documental.

Tabla 2. Aplicación del modelo a los diseños participativos

\begin{tabular}{|c|c|c|c|c|}
\hline \multirow{2}{*}{ Diseños participativos } & \multicolumn{2}{|c|}{ Articulación de la comunidad } & \multicolumn{2}{|c|}{ Incidencia de la comunidad } \\
\cline { 2 - 5 } & $\begin{array}{c}\text { Activación } \\
\text { colectiva }\end{array}$ & $\begin{array}{c}\text { Procesos de } \\
\text { diálogo }\end{array}$ & $\begin{array}{c}\text { Participación } \\
\text { central / periférica }\end{array}$ & $\begin{array}{c}\text { Grado de } \\
\text { influencia }\end{array}$ \\
\hline Participación testimonial & Medio & Bajo & Periférica & Nulo \\
\hline Participación documental & Medio & Bajo & Central / Periférica & Medio \\
\hline Participación dialógica & Alto & Alto & Periférica & Medio \\
\hline Participación generativa & Medio & Bajo & Central / Periférica & Alto \\
\hline Participación expansiva & Alto & Alto & Periférica & Bajo \\
\hline
\end{tabular}

Fuente: Elaboración propia

\section{Conclusiones}

La incidencia que tienen el equipo de producción y la comunidad en la construcción de significado del documental no suele ser equivalente, como se ha indicado. Aunque la dinámica de participación pierde así parte de su capacidad para generar un discurso plural en la obra interactiva, y teniendo en cuenta que la noción tradicional de autoría quedaría casi intacta, en la creación colaborativa con vocación social hay que valorar también la generación de otros efectos positivos para la comunidad. Para ello, es importante tener en cuenta la aplicación y el funcionamiento de distintos diseños participativos, como los que propone esta investigación, ya que esto posibilita una perspectiva amplia y sistemática para la evaluación de la creación colaborativa.

El modelo de análisis que se presenta en este artículo ha valorado, precisamente, la elevada diversidad que adquieren los procesos participativos que se habilitan en los $i$-doc colaborativos. Tras aplicar este modelo, se ha podido comprobar cómo los diseños participativos que lograrían una mayor articulación de la comunidad no son necesariamente los que consiguen una mayor incidencia de la misma en el discurso del documental. Esto se percibe con mayor certeza cuando se compara la participación generativa con los diseños dialógico o expansivo. En el primer caso, encontramos un grado elevado de influencia de la comunidad sobre el contenido del documental. En cambio, sus miembros no contarían con las herramientas suficientes para tener una perspectiva de crecimiento como grupo o para generar una relación productiva. En ese sentido, el beneficio que obtendría la 
comunidad se ve disminuido. En los diseños dialógico y expansivo ocurre lo contrario: una escasa incidencia en el documental no impide que este pueda servir para generar una relación productiva entre quienes participan gracias al diseño del $i$ - $d o c$ colaborativo, que favorece la interrelación constante y la acción colectiva. Así, si bien la influencia de los participantes en la construcción de significado del documental puede tener una cierta correspondencia con su posibilidad para articular acciones colectivas, estos no son elementos coordinados.

Por este motivo, la presente investigación ha demostrado la pertinencia de combinar distintos diseños participativos para establecer un proceso más significativo y más cercano a la noción de participación que se argumentaba al principio de este artículo. Así lo demuestran algunos de los casos que forman parte de la muestra escogida, porque conjugan más de un diseño, como se puede apreciar en iniciativas como (des)iguales, Primal o Proyecto Quipu.

Este artículo aporta así una herramienta para estudiar la participación con un enfoque que parte de la distinción entre la variedad de formas que esta puede adquirir en el $i$-doc colaborativo, con distintas consecuencias para la experiencia del sujeto que participa. Queremos destacar, por último, que aunque se ha elaborado en aplicación a los documentales interactivos en los que contribuye la comunidad en línea, una parte significativa de este modelo es transversal al análisis de otros procesos de creación colaborativa.

\section{Bibliografía.}

Aston, J. y Gaudenzi, S. (2012). Interactive documentary: setting the field. Studies in Documentary Film, 6(2), 125-139. https://dx.doi.org/10.1386/sdf.6.2.125_1

Aston, J. y Odorico, S. (2018). The Poetics and Politics of Polyphony: Towards a Research Method for Interactive Documentary. Alphaville: Journal of Film and Screen Media, 15, 63-93.

Barry, M. y Doherty, G. (2016). What we talk about when we talk about interactivity: Empowerment in public discourse. New Media \& Society. https://doi. org/10.1177/1461444815625944

Carpentier, N. (2011). Contextualising Author-Audience Convergences. Cultural Studies, 25(4-5), 517-533. https://doi.org/10.1080/09502386.2011.600537

Carpentier, N. (2015). Differentiating between access, interaction and participation. Conjunctions. Transdisciplinary Journal of Cultural Participation, 2(2). https://doi.org/10.7146/tjcp.v2i2.23117

Carpentier, N., Duarte, A. y Ribeiro, F. (2019). Rescuing participation: a critique on the dark participation concept. Comunicação e Sociedade, 36, 17-35. https://doi.org/10.17231/ comsoc.36(2019).2341

Cortés-Selva, L. y Pérez-Escolar, M. (2016). Civic participation and interactive documentaries: a contribution to the open government model. El Profesional de la Información, 25(4), 578-587. http://dx.doi.org/10.3145/epi.2016.jul.07

Deuze, M. (2008). Corporate Appropiation of Participatory Culture. En N. Carpentier y S. Livingstone, (Eds.), Participation and Media Production: Critical Reflections on Content Creation (pp. 27-40). Cambridge Scholars Publishers.

Dovey, Jon (2014). Documentary Ecologies: Collaboration and Exploitation. En K. Nash, C. Hight y C. Summerhayes, (Eds.), New Documentary Ecologies. Emerging Platforms, Practices and Discourses (pp. 11-33). Palgrave Macmillan. 
Gaudenzi, S. (2013). The Living Documentary: from representing reality to co-creating reality in digital interactive documentary (Tesis doctoral). University of London, Londres.

Gaudenzi, S. (2014). Strategies of Participation: The Who, What and When of Collaborative Documentaries. En K. Nash, C. Hight y C. Summerhayes, (Eds.), New Documentary Ecologies. Emerging Platforms, Practices and Discourses (pp. 129-148). Palgrave Macmillan.

Gifreu-Castells, A. (2013). El documental interactivo como nuevo género audiovisual. Estudio de la aparición del nuevo género, aproximación a su definición y propuesta de taxonomía y de modelo de análisis a efectos de evaluación, diseño y producción (Tesis doctoral). Universitat Pompeu Fabra, Barcelona.

Gifreu-Castells, A., Sánchez-Castillo, S. y Galán, E. (2019). Aproximación al documental interactivo como formato nativo transmedia. Pasavento. Revista de Estudios Hispánicos, 7(2), 275-302.

Green, D. P., Bowen, S., Hook, J. y Wright, P. (2017). Enabling Polyvocality in Interactive Documentaries through "Structural Participation". Proceedings of the ACM CHI Conference on Human Factors in Computing Systems, 6317-6329. https://doi.org/10.1145/3025453.3025606

Jenkins, H. y Carpentier, N. (2013). Theorizing participatory intensities: A conversation about participation and politics. Convergence, 19(3), 265-286. https://doi.org/10.1177/1354856513482090

Jenkins, H., Ford, S. y Green J. (2013). Spreadable Media: Creating Value and Meaning in a Networked Culture. New York University Press.

Marwick, A. E. (2019). A Cultural History of Web 2.0. En P. Heyer y P. Urquhart, (Eds.), Communication in History. Stone Age Symbols to Social Media (pp. 311-317). Routledge.

Mateos, C. y Gaona, C. (2015). Constantes del videoactivismo en la producción audiovisual. Rastreo histórico (1917-2014) y puntualizaciones para una definición. En F. Sierra y D. Montero, (Eds.), Videoactivismo y movimientos sociales. Teoría y praxis de las multitudes conectadas (pp. 106-137). Editorial Gedisa.

Moreno, V. y Gifreu-Castells, A. (2016). Aproximación al potencial colaborativo de la narrativa documental interactiva en los procesos de cambio social. Cultura, Lenguaje y Representación, 15, 153-169. https://doi.org/10.6035/clr.2016.15.10

Nash, K. (2012). Modes of interactivity: analysing the webdoc. Media, Culture \& Society, 34(2), 195-210. https://doi.org/10.1177/0163443711430758

Nash, K. (2014a). Clicking on the World: Documentary Representation and Interactivity. En K. Nash, C. Hight y C. Summerhayes, (Eds.), New Documentary Ecologies. Emerging Platforms, Practices and Discourses (pp. 50-67). Palgrave Macmillan.

Nash, K. (2014b). Strategies of interaction, questions of meaning: an audience study of the NFBs Bear 71. Studies in Documentary Film, 8(3), 221-234. http://dx.doi.org/10.1080/17503280.2014.9 58904

Nash, K. (2014c). What is interactivity for? The social dimension of web-documentary participation. Continuum: Journal of Media \& Cultural Studies, 28(3), 383-395. http://dx.doi.org/ 10.1080/10304312.2014.893995

Nash, K. (2017). I-docs and the documentary tradition: exploring questions of engagement. En J. Aston, S. Gaudenzi y M. Rose, (Eds.), I-docs. The Evolving Practices of Interactive Documentary (pp. 9-26). Columbia University Press. 
Nichols, B. (1983). The Voice of Documentary. Film Quarterly, 36(3), 17-30. https://doi. org $/ 10.2307 / 3697347$

Odorico, S. (2015). Between Interactivity, Reality and Participation: The Interactive Documentary Form. MEI Mediation and Information, 39, 213-226.

Rose, M. (30 de noviembre de 2011). Four Categories of Collaborative Documentary. Collabdocs. Where Documentary Meets Networked Culture. https://collabdocs.wordpress.com/2011/11/30/ four-categories-of-collaborative-documentary/

Rose, M. (2017a). Documentary as co-creative practice: From Challenge for Change to Highrise - Kat Cizek in conversation with Mandy Rose. En J. Aston, S. Gaudenzi y M. Rose, (Eds.), I-docs. The Evolving Practices of Interactive Documentary (pp. 38-49). Columbia University Press.

Rose, M. (2017b). Not media about, but media with: co-creation for activism. En J. Aston, S. Gaudenzi y M. Rose, (Eds.), I-docs. The Evolving Practices of Interactive Documentary (pp. 4966). Columbia University Press.

Sucari, J. (2020). El documental participativo: visibilidad y experiencia. adComunica. Revista Cientifica de Estrategias, Tendencias e Innovación en Comunicación, (19), 299-302. http://dx.doi. org/10.6035/2174-0992.2020.19.18.

Van Dijck, J. (2009). Users like you? Theorizing agency in user-generated content. Media, Culture \& Society, 31(1), 41-58. https://doi.org/10.1177/0163443708098245

Vázquez-Herrero, J. y Moreno, G. (2017). Documental interactivo iberoamericano: proximidad y transformación social. DOC On-line: Revista Digital de Cinema Documentário, especial, 109130.

Villanueva, S. (2015). Prácticas colaborativas en el documental contemporáneo: propuesta de análisis y revisión del modo participativo en la Teoría del Documental. L'Atalante, 20, 116-123. 